\title{
Estimation of Structural Parameters of the Economic Growth Model With Subspace System Identification
}

\author{
Hiroto Kato, Toshihiko Miyagi \\ Tohoku University, Sendai, Japan
}

\begin{abstract}
The authors are interested in an economic growth model for evaluating the effects of infrastructure investments on the economic growth of a region or a country. This paper proposes a new model based on a real business cycle (RBC) model wherein an increase in human capital contributes to an increase in household intertemporal utility and an increase in infrastructure investment produces an increase in output. Furthermore, the paper proposes a new model—free approach based on the subspace identification with singular value decomposition for estimating the structural parameters included in the RBC model proposed. The basic idea behind the subspace identification method is that the Kalman filter state sequence can be directly estimated from observations by the orthogonal projection of the future output space onto the past output space. The authors used the postwar Japan quarterly data for GDP, consumption, investment, and hours worked between 1970 and 2005. To characterize the subspace-based method they compared the simulation results of the method with those obtained from the hybrid model where a maximum-likelihood method with a Kalman filter is adopted. The subspace-based method demonstrated a high performance in terms of replicating time series data. The authors conclude that system identification is a good tool for linking economic theories and empirical models, and that it compensates for variables omitted from the underlying economic models.
\end{abstract}

Keywords: economic growth model, estimation of structural parameters, subspace identification method, public capital stock

\section{Introduction}

Regional development has become increasingly important as an issue during recent years. In order to achieve autonomous growth of regional economies, it is important to strengthen the productivity and also the intraregional cycles among investment, consumption, and production. It has been widely argued that public infrastructure is one of the important sources of economic growth and enhances the productivity of any regional economy (Aschauer, 1989). However, little work on the economic impact of public investment in connection with other macroeconomic variables has been done so far. The authors are therefore interested in an economic growth model for evaluating the effects of infrastructure investments on the economic growth of a region or country.

The purpose of this paper is twofold: Firstly, the authors propose a variant of the standard real business

Hiroto Kato, Master of Information Sciences, Graduate School of Information Sciences, Tohoku University. Toshihiko Miyagi, Ph.D. of Engineering, Professor, Graduate School of Information Sciences, Tohoku University. Correspondence concerning this article should be addressed to Hiroto Kato, Department of Civil Eng., School of Eng. Tohoku University, Aoba 6-6-06, Aramaki, Aoba-ku, Sendai 980-8579, Japan. E-mail: kato@plan.civil.tohoku.ac.jp. 
cycle model in which an increase in human capital contributes to an increase in household intertemporal utility and an increase in infrastructure investment produces an increase in output; Secondly, the authors propose a new estimation method for the structural parameters included the RBC model. Our parameter-estimation is essentially a model-free approach, based on the subspace identification method with singular value decomposition. The basic idea behind the subspace is that the Kalman filter state sequence can directly be estimated from observations by the orthogonal projection of the future output space onto the past output space. This property enables us to more robustly predict the trajectories of economic growth paths; however, since the state equation is treated as a black box in the system identification method, the structural parameters are not explicitly represented by the estimated coefficients. Therefore, people need an equation system that relates the estimated coefficients of the state equations to the structural parameters in the RBC model.

To identify our RBC model, the authors use the postwar Japan quarterly data for GDP, private consumption, private investment, government consumption, public investment, and hours worked between 1970 and 2005. To analyze the performance of the subspace-based method, the authors compare the simulation results of the proposed method with those obtained from a hybrid model (Ireland, 2004) in which a maximum-likelihood method with a Kalman filter is adopted. The authors show through this comparison that the subspace-based method demonstrates high performance is terms of replicating time series data, that it is a good tool for linking economic theories and empirical models, and that it compensates for variables omitted from the underlying economic models.

\section{Model}

In this section, the authors develop a simple dynamic stochastic general equilibrium model of public investment and public capital stock. The model belongs to a family of traditional RBC models. These RBC models are relatively simple since they do not incorporate the features of New-Keynesian models. But important details are not lost by not using a more elaborate model, because the main objectives are to develop quantitative implications in a simple framework and to estimate structural parameters using system identification.

An infinitely lived representative agent simultaneously plays the roles of household, firm, and government in a single-good, stochastic economy. The representative household derives utility from private consumption $C p_{t}$, government consumption $C g_{t}$, and labor $L_{t}$ during each period as described by the lifetime expected utility function:

$$
E_{0} \sum_{t=0}^{\infty} \rho^{t}\left(\ln C p_{t}+\mu \ln C g_{t}-\tau L_{t}\right)
$$

where $\rho \in(0,1)$ is the discount factor, and $\mu$ and $\tau$ are positive parameters. The indivisible labor can be motivated by assuming that the economy consists of individual consumers, each of whom either works full time or remains unemployed (Hansen, 1985). The representative agent produces output $Y_{t}$ with private capital stock $K_{t}$, human capital stock $H_{t}$, public capital stock $G_{t}$, and labor $L_{t}$ according to the constant returns to scale Cobb-Douglas production function:

$$
Y_{t}=A_{t} K_{t}^{\alpha} H_{t}^{\beta} G_{t}^{\gamma}\left(\eta^{t} L_{t}\right)^{1-\alpha-\beta-\gamma}
$$

where $\eta$ measures the gross rate of labor-augmenting technological progress and the elasticities with output $0<\alpha, \beta, \gamma<1$. The technology shock $A_{t}$ follows the first-order autoregressive process: 


$$
\ln A_{t}=(1-\omega) \ln A+\omega \ln A_{t-1}+\varepsilon_{t}, \quad \varepsilon_{t} \sim N\left(0, \sigma^{2}\right), \operatorname{cov}\left(\varepsilon_{i}, \varepsilon_{j}\right)=0, i \neq j
$$

where $A>0$ is a constant term and $-1<\omega<1$ is the first-order regression parameter. The serially uncorrelated innovation stochastic random term $\varepsilon_{t}$ is normally distributed white noise with standard deviation $\sigma$.

During each period, the representative agent divides output $Y_{t}$ among private consumption $C p_{t}$, government consumption $I p_{t}$, private investment $C g_{t}$, and public investment $I g_{t}$ subject to the budget constraint (4):

$$
Y_{t}=C p_{t}+C g_{t}+I p_{t}+I g_{t}
$$

By investing during period $t$, the representative agent increases the capital stocks $K_{t+1}, H_{t+1}$ and $G_{t+1}$ available during period $t+1$ according to:

$$
\begin{gathered}
K_{t+1}=I p_{t}+\left(1-\delta_{K}\right) K_{t} \\
H_{t+1}=\left(C g_{t}\right)^{\psi_{1}}\left(I g_{t}\right)^{\psi / 2}+\left(1-\delta_{H}\right) H_{t} \\
G_{t+1}=I g_{t}+\left(1-\delta_{G}\right) G_{t}
\end{gathered}
$$

where the depreciation rates satisfy $0<\delta_{K}, \delta_{H}, \delta_{G}<1$. For human capital accumulation, the Uzawa-Lucas (Lucas, 1988) model is representative of models in related research. Gong, Greiner, and Semmler (2004) assumed that the production function of human capital can be considered to have an economy of scale in the Uzawa-Lucas model and use educational expenditure as a proxy variable for human capital. In contrast, the authors introduce government consumption expenditure and public investment to the model and assume diminishing returns to scale in human capital (determined by parameters $\psi_{1}$ and $\psi_{2}$ ).

Equilibrium allocations for this economy solve the representative agent's problem: maximize the utility function (1) subject to constraints (2)-(8) for $t=0,1,2, \ldots$ the authors solve the model by the following standard solution method for the RBC model (Uhlig, 1999):

(1) We derive the equilibrium condition by solving the maximization problem of the representative agent;

(2) We calculate the steady state of the model after dividing each variable by $\eta$;

(3) The non-linear stationary equilibrium conditions can be log-linearized to describe the behavior of the stationary variables as fluctuations around their steady state values in response to random shocks to technology;

(4) Equations of the equilibrium conditions now form a system of linear stochastic difference equations. If we apply the Blanchard-Kahn (1980) condition to the equations, we can write the solution of the model using a state space representation.

After completing the above process, the state space model is finally written as:

$$
\begin{gathered}
s_{t+1}=\Pi(\theta) s_{t}+B \varepsilon_{t+1} \\
f_{t}=U(\theta) s_{t}
\end{gathered}
$$

where $\Pi$ and $U$ are system matrices consisting of elements of the set of structural parameters $\theta$. State vector $s_{t}$, observation vector $f_{t}$, and matrix $B$ are specified as follows:

$$
\begin{aligned}
& s_{t}=\left[\begin{array}{lllll}
k_{t} & h_{t} & g_{t} & a_{t}
\end{array}\right]^{\prime} \\
f_{t}= & {\left[\begin{array}{llllll}
y_{t} & c p_{t} & c g_{t} & i p_{t} & i g_{t} & l_{t}
\end{array}\right]^{\prime} } \\
& B=\left[\begin{array}{lllll}
0 & 0 & 0 & 1
\end{array}\right]^{\prime}
\end{aligned}
$$

The log-linearized variables represent deviations from the steady state. The variables of the state vector are assumed to be the hidden state variables, which are not directly observed. The conventional 
production-function method (Aschauer, 1989) requires capital stock data for estimating the productivity of public capital. However, capital stock data is usually not observed directly, and so instead we use estimated data. This is a significant difference from the conventional production-function method. The method can also handle such variables as human capital stock and technological progress that are difficult to estimate.

\section{Subspace Identification}

Subspace identification is a method for estimating the system matrices of a state space model directly using singular value decomposition (SVD) applied to a block Hankel matrix comprising input-output data. Subspace identification can be easily extended to multiple-input-multiple-output (MIMO) systems and can determine a unique solution; it is applied in various fields because of its high-speed performance. A state space model without input variables or control variables is called a stochastic state space model. Such a model was first proposed by Akaike (1974) and led to the development of related research by Overschee (1996). Determining the stochastic state space model is expressed as an implementation problem of a stochastic system. The implementation problem is, given the input-output data and a finite impulse response sequence, identify the dimensions of the system and the system matrix. Here, the authors can uniquely construct a state space model by using an information matrix called the block Hankel matrix.

\section{State Space Model}

The state space model as a linear dynamic system consists of state equation (13) and observation equation (14), as follows:

$$
\begin{aligned}
& x_{t+1}=A x_{t}+w_{t} \\
& y_{t}=C x_{t}+v_{t}
\end{aligned}
$$

where $x_{t}$ is the state vector, $y_{t}$ is the observation vector, $A$ and $C$ are system matrices, $w_{t}$ and $v_{t}$ are system noises, and $Q$ and $R$ are covariance matrices.

\section{Stochastic Implementation Issue}

Stochastic subspace identification proposed by Faurre (1976) is a method to obtain a Markov representation of the time series or state space model to obtain a time series having a given covariance matrix using the theory of determinate system's implementation problem (Zeiger \& McEwen, 1974) and spectral decomposition.

The authors construct the covariance matrix $\{\Lambda(j), j=0,1, \cdots, L\}$ from the stationary time series $\{y(t), t=0, \pm 1, \cdots\}$ at current time $t$. Finite-dimensional vectors based on past and future time series are defined by (15) and (16).

$$
\begin{aligned}
& p(t)=\left[\begin{array}{llll}
y(t-1) & y(t-2) & \cdots & y(0)
\end{array}\right]^{\prime} \\
& f(t)=\left[\begin{array}{llll}
y(t) & y(t+1) & \cdots & y(L)
\end{array}\right]^{\prime}
\end{aligned}
$$

The authors construct the following cross covariance matrix of the future and past $H_{k k}$ : the block Hankel matrix.

$$
H_{k k}=\left\{f(t) p^{T}(t)\right\}=\left[\begin{array}{cccc}
\Lambda(1) & \Lambda(2) & \cdots & \Lambda(k) \\
\Lambda(2) & \Lambda(3) & \cdots & \Lambda(k+1) \\
\vdots & \vdots & \ddots & \vdots \\
\Lambda(k) & \Lambda(k+1) & \cdots & \Lambda(2 k-1)
\end{array}\right]
$$


Cycle length $K$ determines the size of the block Hankel matrix $H_{k k}$, where $2 k-1 \leq L$, and $k>n$. The authors apply singular value decomposition to $H_{k k}$ to obtain:

$$
H_{k k}=\left[\begin{array}{ll}
U_{s} & U_{n}
\end{array}\right]\left[\begin{array}{cc}
\Sigma_{s} & 0 \\
0 & \Sigma_{n}
\end{array}\right]\left[\begin{array}{c}
V_{s}^{T} \\
V_{n}^{T}
\end{array}\right] \cong U_{s} \Sigma_{s} V_{s}^{T}
$$

where $\Sigma_{n}$ is diagonal matrix arranged the $n$ singular values of $H_{k k}$ in the order of magnitude on the diagonal pieces. Singular values of $\Sigma_{n}$ are sufficiently small. Based on this singular value decomposition, the expanded observability matrix and the expanded reachability matrix are set as follows:

$$
\Gamma_{k}=U_{s} \Sigma_{s}^{1 / 2}, \Omega_{k}=\Sigma_{s}^{1 / 2} V_{s}^{T}
$$

Then, the matrix $A, C$ and $\bar{C}^{T}$ are given by:

$$
A=\underline{\Gamma}_{k}^{\dagger} \bar{\Gamma}_{k}, C=\Gamma_{k}(1: p, 1: n), \bar{C}^{T}=\Gamma_{k}(1: n, 1: p)
$$

where $\underline{\Gamma}_{k}$ and $\bar{\Gamma}_{k}$, respectively, are the matrix obtained by deleting the $p$ th line from the bottom, and that obtained by deleting the $p$ th line from the top. $\dagger$ indicates the Moore-Penrose generalized inverse matrix. We construct the algebraic Ricacci equation (21) using $A, C, \quad \bar{C}$ and $\Lambda(0)$.

$$
\Pi=A \Pi A^{T}+\left(\bar{C}^{T}-A \Pi C^{T}\right)\left(\Lambda(0)-C \Pi C^{T}\right)^{-1}\left(\bar{C}-C \Pi A^{T}\right)
$$

It can calculate the Kalman gain $K$ by using the minimum solution $\Pi^{*} \geq 0$ in the following:

$$
K=\left(\bar{C}^{T}-A \Pi_{*} C^{T}\right)\left(\Lambda(0)-C \Pi_{*} C^{T}\right)^{-1}
$$

Thus, it is possible to configure the state space model from the estimated system matrix and the Kalman gain.

\section{Estimation of the Structural Parameters}

\section{Estimation Method}

The system matrices elements of the state space models (8)-(9), which is a transformed RBC model, are composed of the structural parameters. The state variables are private capital, public capital, human capital, and technological progress, and so the dimension of the system matrix is $n=4$. The dimension of the system matrix in the subspace identification method can, in principal, be any value. The system dimension of interest is the one providing the most stable result. When the system matrices of the state space model have the same dimension as the system matrices estimated by the subspace identification method, the simultaneous equation involving the set the structural parameters as unknown variables can be configured and the value of the structural parameters can be estimated by solving this simultaneous equation.

\section{Results}

Table 1 summarizes the parameter values under the estimation by the subspace identification method. The output elasticity of the capital stocks of the model are measured as $\alpha=0.31, \beta=0.11, \gamma=0.09$. In the subspace identification method as described above, there is no need to provide initial values for the parameters. Therefore, the dependency problem with respect to initial values that occurs when using the Kalman filter is eliminated. In addition to the initial value problem, it has been pointed out for the Kalman filter method that the likelihood function is not necessarily concave (Miyagi, 2009).

\section{Comparison of Forecast Accuracy}

In order to confirm whether predictions given by the subspace identification method replicate the real 
economy, the authors compare them with predictions by the Kalman filter method. In order to do so, the set of observed data from the first quarter of 1970 to the last quarter of 1994 (period 100) is used as the training data set, and the remaining data are used to confirm the forecast accuracy. The target of the forecast is Japanese GDP from period 101.

Table 1

Estimate Values of Structural Parameters

\begin{tabular}{llll}
\hline Parameter & Value & Parameter & Value \\
\hline$\alpha$ & 0.313 & $\delta_{K}$ & 0.0274 \\
$\beta$ & 0.114 & $\delta_{H}$ & 0.0136 \\
$\gamma$ & 0.094 & $\delta_{G}$ & 0.0298 \\
$\mu$ & 0.296 & $A$ & 0.738 \\
$\tau$ & 0.0048 & $\omega$ & 0.944 \\
$\psi_{1}$ & 0.595 & $\sigma$ & 0.0073 \\
$\psi_{2}$ & 0.188 & & \\
\hline
\end{tabular}

In the first stage of the analysis, the system dimension of the subspace identification method is set equal to the dimension of the RBC model, and the cycle length is set to 30 (see Figure 1). By comparing to the predictions obtained using the Kalman filter, it found that the predictions of the subspace identification method capture the trend of the real economy. On the other hand, the smoothed values of the Kalman filter provide more accurate values for the set of training data (see Figure 2). If the cycle length is smaller than 30, short-term reproducibility of prediction increases but long-term predictions tend to deviate significantly. If a sufficiently high system dimension is set, the subspace identification method almost perfectly replicates the real economy. This implies that the potential economic variables that are not included in the RBC model affect the dynamic changes in the data; thus, it suggests incorporation of new economic variables is appropriate. The estimations using the subspace identification method with a larger system dimension than the original economic model are depicted in Figure 3, respectively, for $n=10$ and $n=15$.

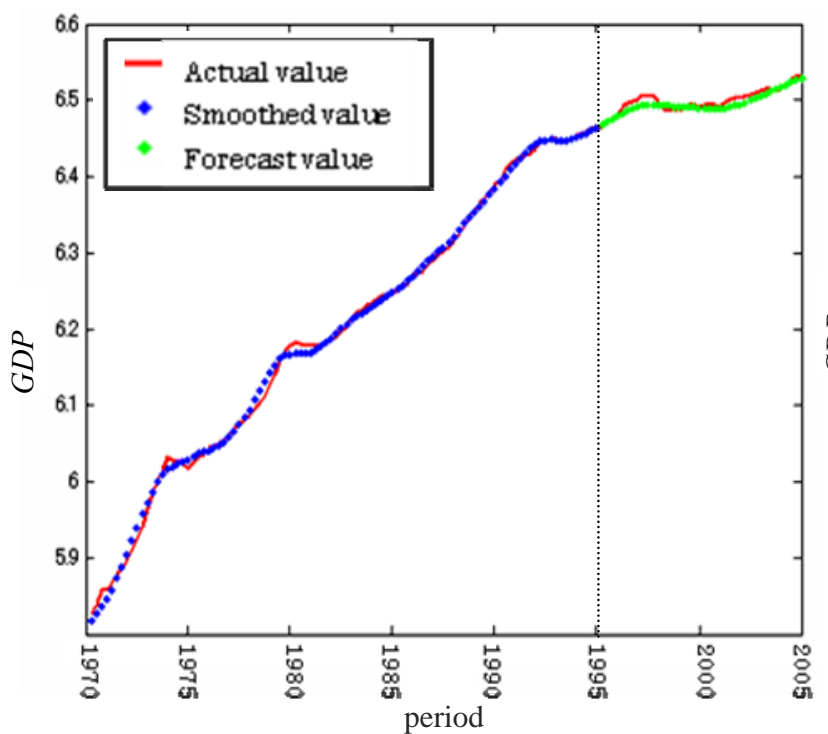

Figure 1. Forecast of GDP by subspace identification.

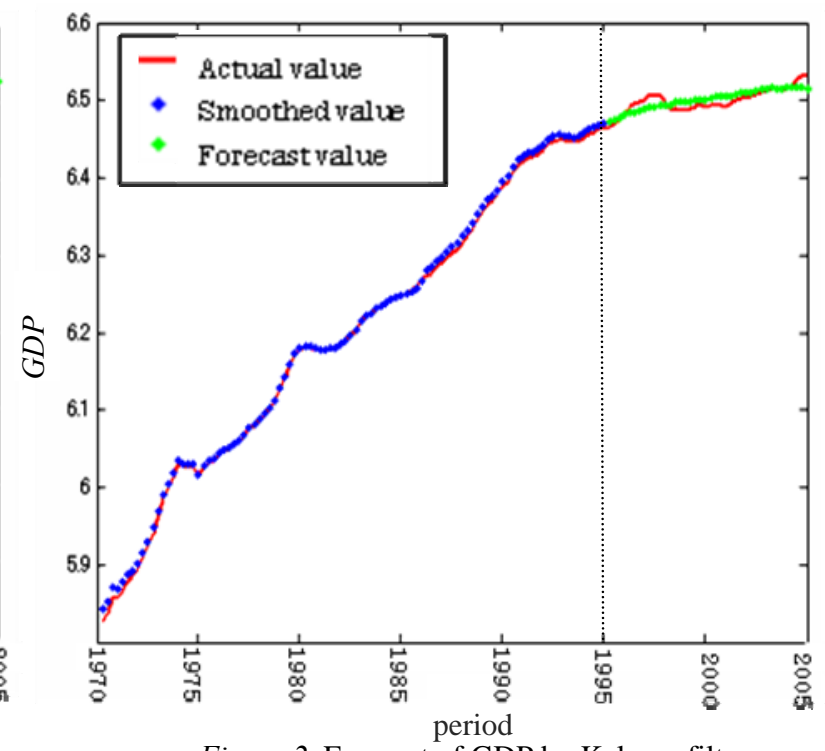

Figure 2. Forecast of GDP by Kalman filter. 


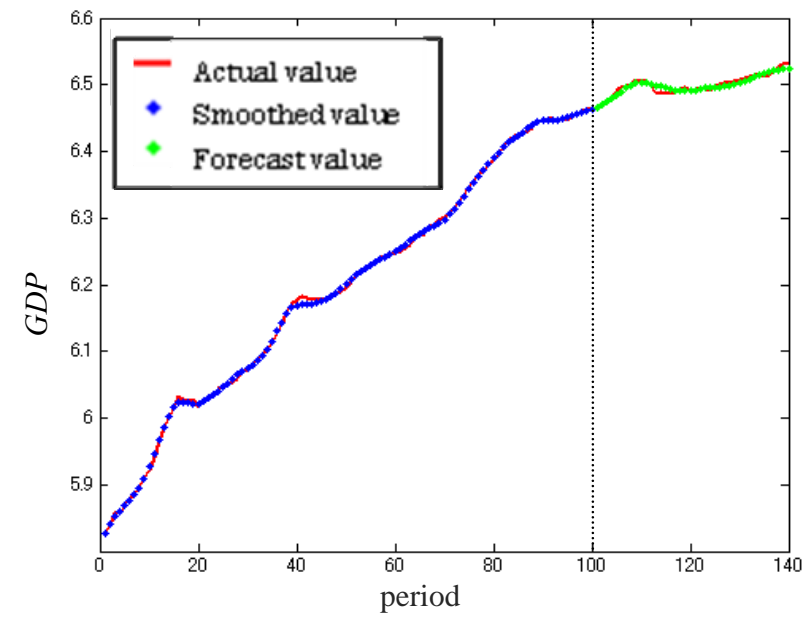

Figure 3. Subspace identification for

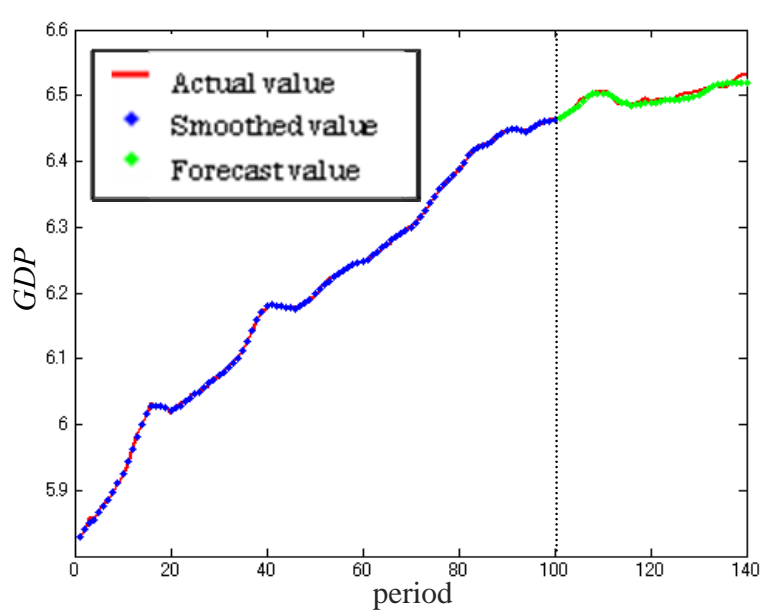

(b)

As a way to improve forecast accuracy, it can consider increasing the amount of available time series data. Sufficient data lead to an exact configuration of the block Hankel matrix and accordingly improves the forecast accuracy. However, the ranges of available macroeconomic time series data are quite limited. Therefore, another option is to increase the number of observation variables in the model itself. Such an increase leads to an increase in the number of corresponding state variables, and so the dimension of the system becomes larger. Since RBC models are very simple structural economic models, they are insufficient for understanding real economic growth. Extending the model with more variables is desirable in terms of forecasting the economy, however, introduction of additional economic variables into the model should have a sound, rigorous economic basis.

\section{Time-Varying Parameter}

When the public capital stock coefficient $\gamma$ in the production function is treated as time-varying parameter, smoothed estimation is as given in Figure 4. As shown, there exists a consistent decreasing trend in the effect of public investment on the Japanese economy. Yoshino and Nakajima (1999) measured Japanese output elasticity and marginal productivity of public capital as a factor of production by using the Cobb-Douglas production-function method. According to their estimations, the output elasticity of public capital during the period 1955-1970 was 0.20, while that during the period 1971-1993 was 0.08 . The result shows that the output elasticity consistently decreased and reached around 0.09 in 1980 and further continued to fall to about 0.086 in 2005. It was not possible to carry out a direct comparison of the results with theirs over the entire time periods because available data for the method began only in 1970. However, there is some similarity between these estimation results. On the other hand, the Policy Research Institute for Land, Infrastructure, Transport, and Tourism (2006) conducted a detail analysis using the panel data of 47 Japanese prefectures and obtained the smaller value of 0.013 as the estimate of output elasticity of the overall public capital stock.

According to the results of the study, the output elasticity is decreasing most quickly in the period 1970-1980. This is consistent with the period for which marginal productivity decreases most swiftly according to Yoshino and Nakajima (1999). System identification enables us to observe a transition of the output elasticity in each quarter. 


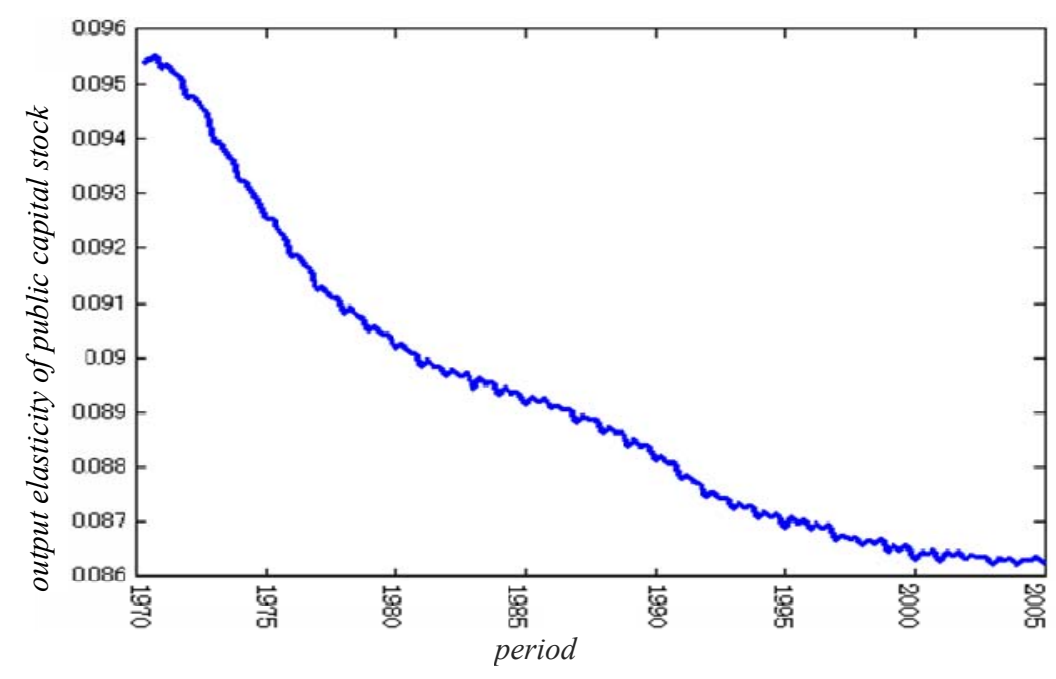

Figure 4. Time series variation of public capital stock’s coefficient $\gamma$.

\section{Conclusion}

The subspace identification method demonstrates strength in long-term forecasting. Forecast accuracy depends on the system dimension and cycle length. The cycle length $k$ used in the model depends on the authors assumptions about economic fluctuations. Here, based on observed data for Japan, it can assumed that cycle length was seven and a half years.

The key ingredient involved in forecast accuracy is the dimension of system matrices. The value set for the system dimension depends on the variables of the economic model. The dimension proposed in this paper is four, but a larger dimension can fit the data and lead to an increase in forecast accuracy. This indicates that other latent variables of the model are affecting the predictions. By using the subspace identification method, it is possible to verify the validity of the proposed model.

In the author's previous study on the productivity effect of public capital (Ashauer, 1989; Yoshino \& Nakajima, 1999), observation data on the public capital stock were used in general, but the model and the method proposed in the current paper treat public capital as a state variable, and there is no need to use such data. Also, the method proposed here can consider random observation error and is a versatile method for estimation of structural parameters (Ireland, 2004). However, on the other hand, the questions on how predictions are affected by using observed public capital stock data, or using quantitative data on physical public infrastructure such as that being developed by Canning (1999) remain.

\section{References}

Akaike, H. (1974). Stochastic theory of minimal realization. IEEE Transactions on Automatic Control, 19(6), 667-674.

Aschauer, D. A. (1989). Is public expenditure productive? Journal of Monetary Economics, 23, 177-200.

Blanchard, O. J., \& Kahn, C. M. (1980). The solution of linear difference models under rational expectations. Econometrica, 48, $1305-1311$.

Canning, D. (1999). Infrastructure’s contribution to aggregate output. Policy research working papers, 2246. World Bank.

Canova, F. (2007). Methods for applied macroeconomic research. New Jersey, USA: Princeton University Press.

Faurre, P. L. (1976). Stochastic realization algorithms. System identification: Advances and case studies (pp. 1-25). New York: Academic Press.

Gong, G., \& Semmlar, W. (2006). Stochastic dynamic macroeconomics: Theory and empirical evidence. New York: Oxford University Press. 
Gong, G., Greiner, A., \& Semmler, W. (2004). The Uzawa-Lucas model without scale effects: Theory and empirical evidence. Structural Change and Economic Dynamics, 15, 401-420.

Hansen, G. D. (1985). Indivisible labor and the business cycle. Journal of Monetary Economics, 16, 309-327.

Ireland, P. N. (2004). A method for taking models to the data. Journal of Economic Dynamics and Control, 24, 1205-1226.

Lucas, R. E. (1988). On the mechanics of economic development. Journal of Monetary Economics, 22, 3-42.

Miyagi, T. (2009). A new framework of dynamic SCGE modeling: A model-free approach using time sequence data. A paper presented at Integration of Spatial Computable General Equilibrium and Transport Modelling, Bilateral Joint Seminar under Agreement between NOW and JSPS, The University of Tokyo.

Overschee, V. P., \& Moor, B. D. (1996). Subspace identification for linear systems. Boston: Kluwer Academic Publisher.

Policy Research Institute for Land, Infrastructure, Transport, and Tourism. (2006). The economic effects of public capital stock: Measuring productivity effects and welfare effects through urban area classification, Report 68.

Uhlig, H. (1999). A toolkit for analyzing nonlinear dynamic stochastic model easily. In R. Marimon, \& A. Scott (Eds.), Computable Methods for the Study of Dynamic Economies (pp. 30-61). New York: Oxford University Press.

Uzawa, H. (1965). Optimum technical change in an aggregate model of economic growth. International Economic Review, 6, 18-31.

Yoshino, N., \& Nakajima, T. (1999). Economic effects of public investment. Tokyo: Nippon-Hyoron-sha.

Zeiger, H. P., \& McEwen, A. J. (1974). Approximate linear realization of given dimension via Ho's algorithm. IEEE Transactions on Automatic Control, 19(2), 153. 